\title{
Evaluación de un programa de formación de profesionales sanitarios sobre abordaje del tabaquismo en pacientes hospitalizados
}

\author{
Montse Ballbè*; Sílvia Mondon**; Gemma Nieva*; Marc Walther**; Esteve Saltó***; Antoni Gual**** \\ *Unidad de Alcohología. Hospital Clínic de Barcelona / Institut Català d’Oncologia. Psicóloga. \\ ** Unidad de Alcohología. Hospital Clínic de Barcelona. Médico psiquiatra. \\ ***DGSP, Departament de Salut. Generalitat de Catalunya. Médico Responsable de Investigación e Información en Educación Sanitaria del Departament de Salut. \\ *** Unidad de Alcohología. Hospital Clínic de Barcelona. Médico psiquiatra. Jefe de la Unidad de Alcohología.
}

\section{RESUMEN}

Introducción: La promoción del abandono del tabaco en el ámbito hospitalario es poco frecuente. Los déficit de formación constituyen una de las principales barreras. Este estudio tiene por objetivo la evaluación de los cambios producidos en relación a conocimientos, actitudes y conductas de los profesionales sanitarios tras recibir formación en intervención mínima. Sujetos y método: Se evalúa la percepción de conocimientos, actuación y status en relación al tabaquismo de 66 profesionales sanitarios antes y después de la formación. Paralelamente se compara la actuación de estos profesionales sobre una muestra de 170 pacientes antes de la formación y otros 170 después de la misma (actuación recibida según los pacientes) con el fin de corroborar la actuación autodeclarada de los profesionales. Resultados: La formación en intervención mínima aumenta significativamente el conocimiento de recursos psicológicos (en un 23,3\%) y farmacológicos (en un $27,1 \%$ ) de los profesionales. Sin embargo, después de la formación no se observan cambios en la medida en que: se pregunta (30,8\% vs. $38,2 \%$, antes y después de la intervención respectivamente); se registra el estatus de fumador de los pacientes $(73,4 \%$ vs. $65,9 \%)$; se inquiere si existe deseo de dejar de fumar $(25 \%$ vs. $12,5 \%)$; o se aconseja el abandono del tabaco (21,9\% vs. $20,8 \%$ ), según la información facilitada por pacientes, y que coincide con la proporcionada por los profesionales. Conclusiones: Es necesario analizar otras barreras que frenan la aplicación de los conocimientos adquiridos.

Palabras clave: Cese del tabaquismo, pacientes internos, educación.

\section{ABSTRACT}

Introduction: The promotion of smoking cessation in hospital settings is uncommon. One of the main barriers to such promotion is lack of knowledge on how to proceed. The objective of this study is to assess changes in professionals' knowledge, attitudes and actions after having received training in brief intervention. Participants and method: Self-perceived knowledge, actions and status in relation to smoking status were assessed in 66 healthcare professionals before and after training. Furthermore, we compared the actions carried out by these professionals with 170 inpatients before the professionals' training and with another 170 after the training (patient's report), to corroborate professionals' self-reported actions. Results: Brief intervention training significantly increased professionals' knowledge of psychological skills (by $23.3 \%$ ) and of pharmacological resources (by $27.1 \%$ ). Nevertheless, changes were not observed in the extent to which professionals: ask (30.8\% vs. $38.2 \%$, before and after the training, respectively); register patients' smoking status (73.4\% vs. $65.9 \%$ ); assess willingness to quit (25\% vs $12.5 \%$ ); or advise patients to quit ( $21.9 \%$ vs. $20.8 \%)$, according to the information provided by patients, which is consistent with that provided by the professionals. Conclusions: It is necessary to examine other barriers to the application of the knowledge gained.

Key words: Smoking cessation, inpatients, education. 


\section{INTRODUCCIÓN}

$\mathbf{L}$ a prevalencia de fumadores en España evoluciona a la baja; no obstante, sigue siendo una de las primeras causas de muerte evitable. Ello justifica los esfuerzos destinados a disminuir el tabaquismo.

La hospitalización constituye una oportunidad óptima para el abandono del tabaco'. La tasa de pacientes ingresados fumadores es elevada, el tabaco ejerce un efecto negativo sobre las intervenciones realizadas durante el ingreso ${ }^{2,3}$, y condiciona mayores costes económicos y más reingresos ${ }^{4}$.

La OMS recomienda la intervención mínima sistematizada en cinco partes (5A) $)^{5}$ : 1) Ask: preguntar sobre el consumo de tabaco, 2) Advice: aconsejar 3) Assess: evaluar 4) Assist: ayudar a dejarlo y 5) Arrange: proponer seguimiento.

Pese a demostrarse la efectividad del tratamiento del tabaquismo en pacientes hospitalizados ${ }^{6}$, la promoción del cese tabáquico en el ámbito hospitalario es poco frecuente. Una de las barreras que explica esta baja frecuencia es la falta de formación $n^{7}$ entre los profesionales sanitarios. El objetivo de este estudio, promovido por la "Xarxa Catalana d'Hospitals Sense Fum" (Red de Hospitales Libres de Humo de Cataluña) consistió en evaluar el impacto de una intervención formativa en los conocimientos, actitudes y conductas de los profesionales sanitarios de un hospital general. Asimismo, este estudio representa la continuación de un trabajo previo de nuestro grupo en donde se averiguaba en qué medida la intervención mínima antitabáquica formaba parte de la rutina de dichos profesionales sanitarios ${ }^{8}$.

\section{MÉTODOS}

\section{Sujetos}

Profesionales: 66 profesionales de cinco unidades del Hospital Clínic de Barcelona (ginecología, obstetricia, medicina interna, traumatología y psiquiatría) que acudieron voluntariamente a las sesiones de formación. La edad media fue de 51,4 años (ds=38,5), el $33,3 \%$ eran médicos, el $48,5 \%$ enfermeros/as, y el resto otros profesionales sanitarios. La muestra para la evaluación después de la formación (los mismos profesionales evaluados antes de la formación) se redujo a 59 debido a la pérdida de 7 profesionales.

Pacientes: 170 pacientes en la evaluación previa a la formación y otros 170 en la posterior, ingresados en las unidades de hospitalización anteriormente descritas, que aceptaron participar voluntariamente en el estudio. En la muestra pre-formación, la edad media fue de 38,94 años $(d s=11,3$ ) y el $77,1 \%$ eran mujeres.
No se encontraron diferencias estadísticamente significativas entre la muestra pre y post formación. Se excluyeron exclusivamente los pacientes con deterioro de funciones cognitivas.

\section{Instrumentos}

Cuestionario autoadministrado para profesionales: Se les interrogaba acerca de la frecuencia con que actuaban en base a las cinco acciones antes mencionadas (5A) cuando los pacientes presentaban patología relacionada con el tabaco y cuando no la presentaban. Finalmente, se les preguntaba por su propio consumo y actitudes en relación al tabaco.

Cuestionario autoadministrado para pacientes: Se les preguntó, en el momento del alta, si habían recibido cada uno de los pasos de la intervención mínima (5A) en el curso de la hospitalización. También se comprobó si en el informe de alta de los pacientes constaba el estatus en relación al tabaco o algún consejo o propuesta de seguimiento.

\section{Procedimiento}

Se evaluó antes y 4 semanas después de la formación a 66 profesionales y a 170 pacientes ingresados en el momento del alta con el fin de corroborar la actuación autodeclarada de los profesionales. La intervención formativa fue de una hora de duración sobre el consumo de tabaco y se impartieron los siguientes temas: a) epidemiología, b) importancia de la intervención en pacientes hospitalizados, c) intervenciones eficaces, d) intervención mínima sistematizada en cinco partes (5A) recomendada por la OMS ${ }^{18:}$ 1) Ask: preguntar sobre el consumo de tabaco, 2) Advice: aconsejar, 3) Assess: evaluar, 4) Assist: ayudar a dejarlo y 5) Arrange: proponer seguimiento, y e) tratamiento farmacológico y estrategias psicológicas.

Se entregó material escrito de recordatorio (folletos para profesionales y posters para las salas) sobre cómo realizar la intervención breve.

Se ofrecía además la posibilidad de dispensar a los pacientes terapia sustitutiva de nicotina gratuitamente durante el ingreso y seguimiento telefónico al mes y a los 6 meses.

La sesión tuvo lugar en un horario accesible al turno de trabajo de mañana y tarde y se entregaron diplomas acreditados por la Comisión de Formación Continuada del Sistema Nacional de Salud y el Consell Català de Formació Mèdica Continuada a los asistentes.

El $54,1 \%$ de los profesionales que trabajaban en las unidades acudió a la formación; el 83,3\% de los médicos y el $47 \%$ de las enfermeras. 


\section{Análisis de datos}

El análisis estadístico de los datos obtenidos de los cuestionarios se realizó mediante el paquete estadístico SPSS (versión 12.0). Para las variables cuantitativas se utilizaron pruebas T. Para las cualitativas, se realizaron pruebas de Chi cuadrado.

Se consideró válida la anotación de "no presenta hábitos tóxicos" en el informe de alta como registro del estatus en relación al tabaco de los pacientes.

\section{RESULTADOS}

Según los resultados basales obtenidos de los cuestionarios de los pacientes, de la muestra de fumadores, un 29,5\% declaraba querer dejar de fumar tras el alta hospitalaria. Sin embargo, sólo un 30,8\% de ellos recordaba haber sido preguntado por su estatus en relación al tabaco, sólo un 21,9\% refería haber recibido el consejo de dejar de fumar, y sólo un $25 \%$ haber sido preguntado acerca de si quería dejar de fumar ${ }^{8}$. Después de la formación de profesionales, estos porcentajes no experimentaron un aumento significativo (ver tabla1).

Al comprobar si los profesionales anotaban el estatus de los pacientes en relación al tabaco en el informe de alta, ello sucedía en un $73,4 \%$ de los casos pero en ningún caso se registraba el ofrecimiento de consejo antitabáquico o seguimiento. Estos porcentajes no experimentaron variaciones significativas tras la formación.

En los cuestionarios de profesionales, se observó que la sesión de formación conseguía el objetivo de aumentar significativamente la percepción de un mayor conocimiento de las estrategias farmacológicas: de un $28,8 \%$ de profesionales que refería tener un conocimiento bueno o bastante bueno, se pasó a un 57,1\% ( $\left.X^{2}=9,448 ; p=0,002 ; g l=1\right)$; y de las herramientas psicológicas: de un $25,8 \%$ a un $50 \%\left(\mathrm{X}^{2}=\right.$ 7,331; $p=0,007 ; g l=1)$.

No se observaron diferencias significativas en las variables relativas a Ask, Advise, Assess, Assist y Arrange, ni en el porcentaje en que los profesionales referían anotar el estatus de los pacientes en relación al tabaco antes y después de la formación (ver tabla 2).

Tabla 1. Intervención mínima recibida, según pacientes.

\begin{tabular}{|l|c|c|c|}
\hline \multicolumn{1}{|c|}{$\mathbf{5 A}$} & $\begin{array}{c}\text { PRE } \\
\text { (antes de la formación) }\end{array}$ & $\begin{array}{c}\text { POST } \\
\text { (después de la formación) }\end{array}$ & Significación \\
\hline Ask ${ }^{1}$ (preguntar) & $30,8 \%$ & $38,2 \%$ & $X^{2}=2,090 ; p=0,148 ; g l=1$ \\
\hline Advise (aconsejar) & $21,9 \%$ & $20,8 \%$ & $X^{2}=0,012 ; p=0,911 ; g l=1$ \\
\hline Assess $^{2}$ (evaluar) & $25,0 \%$ & $12,5 \%$ & $X^{2}=2,078 ; p=0,149 ; g l=1$ \\
\hline
\end{tabular}

'Muestra total de pacientes. Pre-formación, n=170; Post-formación, $n=170$

${ }^{2}$ Muestra de pacientes fumadores. Pre-formación, $n=32$; Post-formación, $n=48$

No se pudo analizar Assist y Arrange por insuficiente tamaño de la n.

Tabla 2. Porcentaje para las respuestas “siempre" o "frecuentemente", según los profesionales.

\begin{tabular}{|c|c|c|c|c|c|c|}
\hline \multirow{2}{*}{$5 \mathrm{~A}$} & \multicolumn{3}{|c|}{ Cuando el paciente sufre patología relacionada con el tabaco } & \multicolumn{3}{|c|}{ Cuando el paciente no sufre patología relacionada con el tabace } \\
\hline & $\begin{array}{l}\text { PRE. Antes de } \\
\text { la formación }\end{array}$ & $\begin{array}{l}\text { POST. Después } \\
\text { de la formación }\end{array}$ & Significación & $\begin{array}{l}\text { PRE. Antes de } \\
\text { la formación }\end{array}$ & $\begin{array}{l}\text { POST. Después } \\
\text { de la formación }\end{array}$ & Significación \\
\hline $\begin{array}{l}\text { Ask } \\
\text { (preguntar) }\end{array}$ & $53,1 \%$ & $61,4 \%$ & $\begin{array}{c}X^{2}=1,069 \\
p=0,586 ; g \mid=2\end{array}$ & $39,7 \%$ & $45,8 \%$ & $\begin{array}{c}X^{2}=1,662 \\
p=0,436 ; g \mid=2\end{array}$ \\
\hline $\begin{array}{l}\text { Advise } \\
\text { (aconsejar) }\end{array}$ & $75,4 \%$ & $69,0 \%$ & $\begin{array}{c}X^{2}=0,696 \\
p=0,706 ; g \mid=2\end{array}$ & $31,3 \%$ & $27,1 \%$ & $\begin{array}{c}X^{2}=0,269 \\
p=0,874 ; g l=2\end{array}$ \\
\hline $\begin{array}{l}\text { Assess } \\
\text { (evaluar) }\end{array}$ & $43,1 \%$ & $49,1 \%$ & $\begin{array}{c}X^{2}=0,568 \\
p=0,753 ; g l=2\end{array}$ & $17,2 \%$ & $17,2 \%$ & $\begin{array}{c}X^{2}=1,451 \\
p=0,484 ; g \mid=2\end{array}$ \\
\hline $\begin{array}{l}\text { Assist } \\
\text { (ayudar) }\end{array}$ & $24,6 \%$ & $34,5 \%$ & $\begin{array}{c}X^{2}=6,250 \\
p=0,044^{*} ; g l=2\end{array}$ & $10,9 \%$ & $17,5 \%$ & $\begin{array}{c}X^{2}=3,269 \\
p=0,195 ; g l=2\end{array}$ \\
\hline $\begin{array}{l}\text { Arrange } \\
\text { (acordar seguimiento) }\end{array}$ & $40,0 \%$ & $51,7 \%$ & $\begin{array}{c}X^{2}=2,226 \\
p=0,328 ; g \mid=2\end{array}$ & $9,4 \%$ & $13,8 \%$ & $\begin{array}{c}X^{2}=1,409 \\
p=0,494 ; g \mid=2\end{array}$ \\
\hline $\begin{array}{l}\text { Registrar en informe } \\
\text { de alta }\end{array}$ & $53,1 \%$ & $61,4 \%$ & $\begin{array}{c}X^{2}=1,058 \\
p=0,589 ; g \mid=2\end{array}$ & $38,1 \%$ & $39,7 \%$ & $\begin{array}{c}X^{2}=0,226 \\
p=0,893 ; g \mid=2\end{array}$ \\
\hline
\end{tabular}

Pre-formación, $n=66$ y Post-formación $n=59$

*Diferencias significativas en los porcentajes para las respuestas de "a veces" y "raramente o nunca" 
Se constató asimismo que el porcentaje de actuación (5A) de los profesionales era significativamente menor cuando el paciente fumador no sufría ninguna patología relacionada con el tabaco, con $p<0,01 \quad(g \mid=1)$ para las 5A.

No se observaron diferencias significativas en la actuación de los profesionales según su estatus frente al tabaco (fumadores frente no fumadores o exfumadores).

Un 75\% de los profesionales consideraba que su acción modélica era importante o muy importante, cifra que no aumentaba significativamente después de la formación. Por otra parte, el grado en que los profesionales que fumaban $(35,4 \%)$ expresaban su intención de dejar de fumar en los próximos seis meses experimentó una tendencia a la mejora (47,6\% basal; 61,9\% post-formación), si bien, ésta no alcanzó la significación estadística.

\section{DISCUSIÓN}

Los resultados obtenidos en los cuestionarios de pacientes (intervención recibida) son congruentes con los obtenidos en los de profesionales (intervención realizada).

Una formación breve en intervención mínima resultó útil para aumentar el conocimiento percibido por los profesionales acerca de los recursos farmacológicos y herramientas psicológicas que existen para abordar el problema del tabaquismo. No obstante, este aumento de conocimientos no comportó una mayor promoción del abandono del tabaco. Además, aunque la mayoría de profesionales consideraba muy importante su acción como modelo, no fue posible modificar, a corto plazo, las actitudes de los numerosos profesionales fumadores en relación a su propio consumo, es decir, disminuirlo o proponerse abandonarlo en los meses próximos evidenciando la necesidad de crear y llevar a cabo programas específicos de tratamiento y/o motivación para los profesionales.

La baja frecuencia detectada en este estudio en lo que atañe a la promoción del abandono del tabaco en el ámbito hospitalario es similar a la documentada en la literatura ${ }^{9}$. Igualmente congruente con la misma es el hallazgo de una frecuencia incluso menor cuando los pacientes no sufren patologías relacionadas con el tabaco ${ }^{9,10}$. De ello se desprende que la actividad asistencial en las unidades de hospitalización especializadas tiende a centrarse en el tratamiento de patologías concretas, dejando en segundo término las intervenciones preventivas. Muchos profesionales, incluso no realizan intervención antitabáquica en sus pacientes aunque éstos sufran alguna patología relacionada con el tabaco. El bajo porcentaje de profesionales que acu- dió a la formación es una muestra de la dificultad para promover actividades preventivas en el ámbito hospitalario y a su vez esto impide saber hasta que punto la ausencia de cambios se debe a que la intervención formativa no fue efectiva o a que casi la mitad de los profesionales no acudieron a realizarla.

Aunque la falta de formación es sin duda una barrera importante a la hora de promover el cese tabáquico en pacientes hospitalizados, nuestro estudio sugiere que el aumento de conocimientos y habilidades por si solo es insuficiente para promover cambios en la rutina asistencial. Nuestra experiencia apunta a la dificultad para implementar actividades preventivas en el ámbito hospitalario como primera barrera a tener en cuenta.

La introducción de nuevas rutinas en la práctica diaria resulta difícil y lenta. A pesar de ello, los esfuerzos destinados a la promoción del abandono del tabaco en los pacientes deben ser continuados, dada la elevada morbimortalidad asociada al consumo.

\section{AGRADECIMIENTOS}

Este estudio ha sido realizado y financiado parcialmente en el marco del convenio de colaboración entre el Instituto Catalán de Oncología (ICO) y la Dirección General de Salut Pública del Departament de Salut para el desarrollo de la Red de Hospitales Sin Humo de Catalunya.

Agradecemos la colaboración del personal sanitario de las unidades de hospitalización citadas del Hospital Clínic de Barcelona.

\section{REFERENCIAS}

1 Fiore MC, Bailey WC, Cohen SJ, Dorfman SF, Goldstein MG, Gritz ER, et al. Treating Tobacco Use and Dependence. Clinical Practice Guideline. Rockville: U.S. Public Health Service 2000.

2 Moller AM, Villebro, N, Pedersen, T, Tonnesen, H. Effect of preoperative smoking intervention on postoperative complications: a randomised clinical trial. Lancet 2002; 359: 114-7.

3 Lavernia CJ, Sierra RJ, Gómez-Marin, O. Smoking and joint replacement: resource consumption and short-term outcome. Clin Orthop Relat Res 1999; 367: 172-80.

4 Hurley SF. Hospitalisation and costs attributable to tobacco smoking in Australia: 2001-2002. Med J Aust 2006; 184: 45. 
5 OMS. Evidence based core recommendations for health care systems in Europe. Partnership Project to Reduce Tobacco Dependence. February; 2001.

6 Rigotti NA, Munafo MR, Murphy MF, Stead LK. Interventions for smoking cessation in hospitalized patients (Cochrane Review). Cochrane Database of Syst Rev 2003; issue 1.

7 Twardella $\mathrm{D}$, Brenner $\mathrm{H}$. Lack of training as a central barrier to the promotion of smoking cessation: a survey among general practitioners in Germany. Eur J Public Health 2005; 15: 140-145.
8 Nieva G, Gual A, Mondon S, Walter M, Saltó E. Evaluación de la intervención mínima en tabaquismo en el ámbito hospitalario. Med Clin (Barc) 2007; 128: 730-2.

9 Miller NH, Smith PM, DeBusk RF, Sobel DS, Taylor CB. Smoking cessation in hospitalized patients: results of randomized trial. Arch Intern Med 1997; 157: 409-15.

10 Goldstein MG, Niaura, C. Willey-Lessne J, DePue C, Eaton W, Rakowski, W et al. Physicians counseling smokers. A population-based survey of patient's perceptions of health care provider-delivered smoking cessation interventions. Arch Intern Med 1997; 157: 1 
